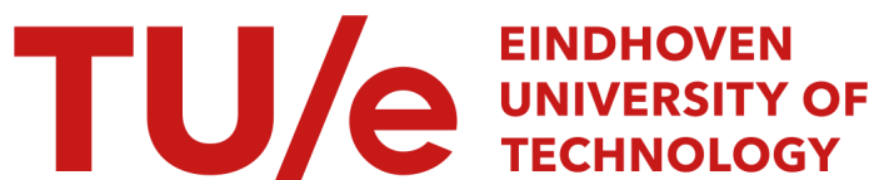

\section{On the propagation analysis of electrohysterographic signals}

\section{Citation for published version (APA):}

Rabotti, C., Mischi, M., Laar, van, J. O. E. H., Oei, S. G., \& Bergmans, J. W. M. (2008). On the propagation analysis of electrohysterographic signals. In EMBS 2008. 30th Annual International Conference of the IEEE Engineering in Medicine and Biology Society, 2008. 20-25 August, 2008, Vancouver, British Columbia, Canada (pp. 3868-3871). Institute of Electrical and Electronics Engineers. https://doi.org/10.1109/IEMBS.2008.4650054

DOI:

10.1109/IEMBS.2008.4650054

Document status and date:

Published: 01/01/2008

\section{Document Version:}

Publisher's PDF, also known as Version of Record (includes final page, issue and volume numbers)

\section{Please check the document version of this publication:}

- A submitted manuscript is the version of the article upon submission and before peer-review. There can be important differences between the submitted version and the official published version of record. People interested in the research are advised to contact the author for the final version of the publication, or visit the $\mathrm{DOI}$ to the publisher's website.

- The final author version and the galley proof are versions of the publication after peer review.

- The final published version features the final layout of the paper including the volume, issue and page numbers.

Link to publication

\section{General rights}

Copyright and moral rights for the publications made accessible in the public portal are retained by the authors and/or other copyright owners and it is a condition of accessing publications that users recognise and abide by the legal requirements associated with these rights.

- Users may download and print one copy of any publication from the public portal for the purpose of private study or research.

- You may not further distribute the material or use it for any profit-making activity or commercial gain

- You may freely distribute the URL identifying the publication in the public portal.

If the publication is distributed under the terms of Article $25 \mathrm{fa}$ of the Dutch Copyright Act, indicated by the "Taverne" license above, please follow below link for the End User Agreement:

www.tue.nl/taverne

Take down policy

If you believe that this document breaches copyright please contact us at:

openaccess@tue.nl

providing details and we will investigate your claim. 


\title{
On the propagation analysis of electrohysterographic signals
}

\author{
Chiara Rabotti, Massimo Mischi, Judith van Laar, Guid Oei, and Jan Bergmans, Senior Member IEEE
}

\begin{abstract}
Premature birth is a leading cause of fetal mortality and long-term morbidity. The effective treatment of preterm uterine contractions requires new methods for predicting delivery. The electrohysterographic (EHG) signal is a measure of the bioelectrical process underlying the uterine contraction. The analysis of parameters derived from the EHG signal can therefore provide fundamental information for the prognosis of labor. In this paper, we focus on the propagation of the EHG signal recorded during delivery by multiple electrodes. For the inter-electrode delay assessment and propagation analysis, two different methods are implemented. One is based on the prior estimation of the uterine mechanical activity by EHG signal processing. The delay is then calculated by the cross-correlation function between the mechanical activity estimated at each sensor. The other method is a high temporal resolution adaptive delay estimator that operates directly on EHG signals. The previously demonstrated accuracy of the mechanical estimates and the agreement between the delays provided by the methods confirm the tight relationship between the mechanical and electrical activity of the uterus. However, our results suggest that a higher temporal resolution delay estimator is preferred.
\end{abstract}

\section{INTRODUCTION}

Preterm birth, which is defined as a birth occurring before 37 completed weeks of gestation, is the potential cause of severe complications [1][2]. The first sign of preterm labor is symptomatic preterm uterine activity in the form of uterine contractions. For suppressing preterm uterine activity, gynecologists resort to tocolytic agents. However, an early therapy is determinant for its effectiveness [2]. Therefore, timely recognition of the process leading to active labor is of prime importance. Nevertheless, there is no technique for objectively monitoring the uterus and assessing whether the organ has entered a state of increased activity and responsiveness that may influence the treatment [2].

The devices and methods currently in use, in fact, do not provide accurate prognosis of preterm labor. Intrauterine pressure catheters are limited by their invasiveness and the need for ruptured membranes. External uterine monitors, such as tocodynamometers, are uncomfortable, and often provide noisy signal of difficult interpretation [2]. Biological tests, such as fetal fibronectin, provide better prognostic capability, but a convincing statistical validation is lacking [2].

This work was supported by the Dutch Technology Foundation STW.

C. Rabotti is with the Department of Electrical Engineering, Eindhoven University of Technology, Eindhoven, the Netherlands. C.Rabottidtue.nl

M. Mischi is with the Department of Electrical Engineering, Eindhoven University of Technology, Eindhoven, the Netherlands.

J. van Laar is with the Department of Obstetrics and Gynecology, Máxima Medical Center, Veldhoven, the Netherlands.

G. Oei is with the Department of Obstetrics and Gynecology, Máxima Medical Center, Veldhoven, the Netherlands.

J. Bergmans is with the Department of Electrical Engineering, Eindhoven University of Technology, Eindhoven, the Netherlands.
Even cervical changes may not be an accurate indicator of true labor, as a large percentage of women with established cervical change do not deliver preterm even when not treated with tocolytics [1].

The electrohysterogram (EHG) is the signal associated to the electrical activity propagating through the myometrium cells during a contraction. In particular, the EHG signal is the primary cause of the mechanical uterine activity; therefore, it is potentially the best predictor of delivery.

In the literature, several studies investigated the use of the EHG signal for predicting labor and distinguishing preterm contraction ultimately leading to preterm delivery. Overall, many parameters derived from the EHG signal have been considered, in both time [3] and frequency domain [4][5]. The shift of the EHG burst frequency components from low frequencies, during pregnancy, to higher frequencies, during labor, seems the most significant and earliest observable variation, experienced in both term and pre-term delivery [5].

Nevertheless, cut-off values of frequency for an accurate prognosis of labor are not yet available. Further investigations need to address the prognostic value of other EHG parameters that may be representative of the uterine functionality.

The spreading of electrical activity in the myometrium is the first trigger of a coordinated and effective contraction; a multi-channel analysis of direction and speed of the propagating EHG signal can then provide a fundamental contribution for predicting delivery. To this end, the first step is the calculation of the relative time-lags among the burst recordings at different sensor locations.

Previously, we proposed a method for inter-electrode delay assessment based on the prior estimation of the internal uterine pressure (IUP) from multichannel EHG recordings [6]. The delays were calculated by finding the maximum value of the cross-correlation function (CCF) among the IUP estimates in the different channels. In general, the accuracy of the delays obtained by CCF maximization may be hampered by poor inter-channel correlation. Noteworthy, the high correlation among the IUP estimates in the different channels ensures more robustness with respect to poorly correlated EHG signals.

In this paper, we compare the method based on the IUP estimates with a high time-resolution adaptive delay estimator operating on EHG signals rather than IUP estimates. The methods are evaluated on real signals recorded by 8 electrodes on one woman. The recording covers the last 5 hours of delivery and includes 110 contractions. 


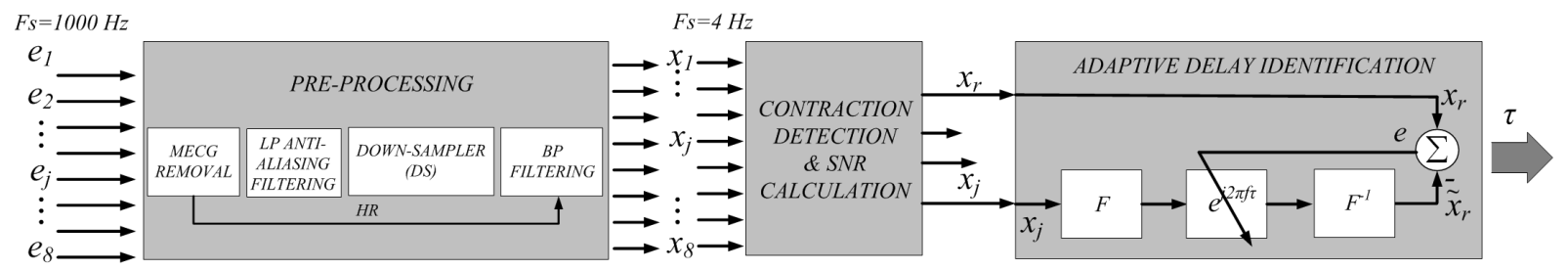

Fig. 1. Algorithm scheme of the adaptive delay estimator. The recorded unipolar EHG signals, $\left(e_{1}, \ldots, e_{8}\right)$ are preprocessed to improve the SNR: after MECG removal and low pass (LP) anti-aliasing filtering, the sampling frequency $\left(F_{s}\right)$ is reduced to $4 \mathrm{~Hz}$ by a down-sampler (DS) and the signals are band-pass (BP) filtered. The higher cut-off frequency of the BP filter is automatically set depending on the mother's HR. To single-out the reference electrode signal $x_{r}$ for the adaptive delay estimator, the second block comprises the detection of contractions and calculation of the signal-to-noise ratio (SNR). The delay $\tau$ is then adaptively identified for each electrode signal $x_{j}$ in the frequency domain.

\section{Methodology}

The propagation analysis of the EHG signal was performed using the method in [6] and an adaptive delay estimator, implemented in the frequency domain to obtain a higher temporal resolution. The method in [6] comprises the prior estimation of the IUP from the EHG signal in each electrode. The inter-electrode delay is then assessed by locating the maximum of the CCF between the IUP estimated at each electrode $j$ and the IUP estimate at the electrode $r$, i.e., the sensor with the best SNR. The method for the high resolution delay assessment is schematically represented in Fig. 1 and more accurately described in the following. The preprocessing and the calculation of the reference electrode differ for the two methods only for the frequency of the down-sampler (DS), which is $10 \mathrm{~Hz}$ for the IUP-estimatebased method and $4 \mathrm{~Hz}$ for adaptive delay estimator.

\section{A. Data acquisition}

The experimental data were collected at the Máxima Medical Center in Veldhoven (the Netherlands). After accurate skin preparation for skin impedance reduction, eight disposable contact $\mathrm{Ag}-\mathrm{AgCl}$ electrodes were placed on the abdomen (10-15 cm inter-electrode distance) as reported in [6]. The common reference for the recording electrodes was placed on the right hip. In order to obtain an efficient rejection of the electromagnetic interference, we employed a driven-right-leg ground electrode and actively shielded cables. The IUP was simultaneously measured by a Koala M1333A (Philips Medical Systems, Best, the Netherlands) intrauterine pressure catheter inserted in the uterine cavity due to medical prescription. The EHG signals were recorded at $1000 \mathrm{~Hz}$ and digitized at 20-bit resolution by an M-PAQ amplifier (Maastricht Instruments Ltd., the Netherlands), a system for physiological measurement. We analyzed the signal recorded on one woman until the end of delivery. In total, we analyzed approximately 5 hours of recording containing 110 uterine contractions.

\section{B. Pre-processing}

The uterine EHG signal can be affected by several noise sources, e. g., ECG signals, electromyographic (EMG) interference generated by the abdominal skeletal muscles, and several motion artifacts. Part of this noise can be reduced by employing bipolar recording. Unfortunately, for the analysis of action potential propagation in the myometrium, unipolar measurements are preferred. An accurate pre-processing of the signals for suppressing the main interferences was therefore necessary.

One of the main interferences in unipolar EHG recordings is the MECG. Therefore, the method described in [7] was adopted for the MECG removal. The baseline oscillations due to slow electrical waves, such as respiration, were then removed by a four-order Butterworth filter with cut-off frequency set at $0.34 \mathrm{~Hz}$ [4].

Unfortunately, some oscillations occurring at the maternal heart-rate (HR), which are not present when bipolar configurations are used, are still visible in the unipolar EHG signal after MECG removal. A low-pass filter was then employed with cut-off frequency automatically chosen on the basis of the calculated mother's HR. In particular, the stop band of the filter was set at the minimum value of the instantaneous maternal HR. In order to obtain a steep transitional band, we used a Chebyshev Type II filter with the minimum order required to meet the following specification: $0.4 \mathrm{~Hz}$ transition bandwidth, $3 \mathrm{~dB}$ maximum pass-band ripple, and $60 \mathrm{~dB}$ stopband attenuation. The signal was then confined in a narrow band that is upper bounded by the minimum value of HR, which is lower than $2 \mathrm{~Hz}$. The sampling rate could therefore be decreased up to $4 \mathrm{~Hz}$. As a decrease of the sampling frequency reduces significantly the computational time, a down-sampler (DS) was employed, after the MECG removal and after low-pass anti-aliasing filtering.

The electrical signal associated to voluntary contraction of the abdominal muscle has a dominant frequency component of about $30 \mathrm{~Hz}$ [3]. After low-pass filtering, the noise due to EMG activity is therefore negligible.

\section{Adaptive delay estimator}

The uterine contractions were detected and separated to single-out the signal $x_{r}$ with the best SNR. We defined the SNR as the ratio between the power of the signal during a contraction and the power during the quiescent period [8]. The signal of the electrode $r$ was then used as the reference for the delay calculation. For each contraction segment and electrode signal $x_{j}$, the delay $\tau$ was then adaptively estimated. By implementing the estimator in the frequency domain a delay $\tau$ with unlimited temporal resolution can be obtained. 
When the Fourier transform is applied to time-translations of the same signal the phase difference of the two Fourier transformed signals is, in fact, a linear function of the frequency. The continuous time delay, $\tau$, between the signals corresponds to the slope of the straight line representing the signal phase difference as a function of frequency.

The adaptive delay estimator was then based on a Simplex Search method [9] implemented in the frequency domain using the discrete Fourier transform (DFT) matrix F.

\section{RESULTS}

The Bland-Altman plot [10] of the delay estimates provided by the analyzed methods is reported in Fig. 2, where $\tau_{I U P}$ is the delay obtained by the IUP estimates, and $\tau$ that obtained by the adaptive delay estimator. From the plot the agreement between the values of delay calculated by the two methods can be easily assessed. The average absolute difference between the delays, was 0.32 seconds, which is larger than the maximum resolution of the delay calculated by the CCF at $10 \mathrm{~Hz}$. The standard deviation of the difference was equal to $0.8 \mathrm{~s}$.

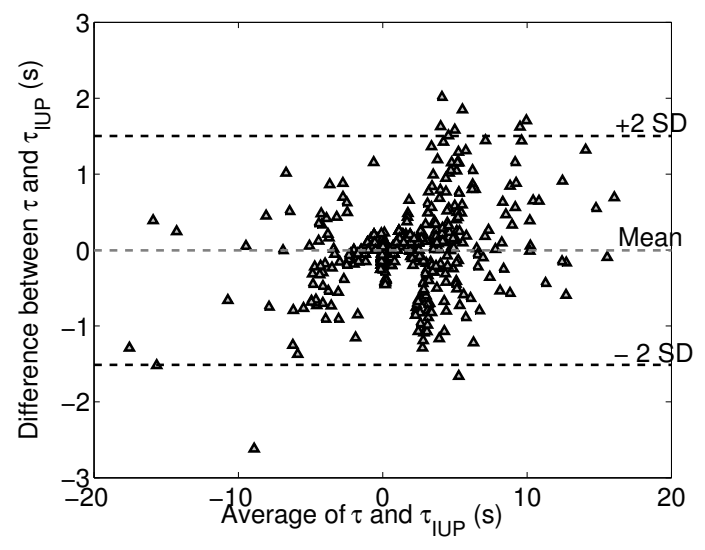

Fig. 2. Bland-Altman plot of the delay calculated by the two algorithms.

The relative detection delays of the electrodes during the same contraction allowed an estimate of the direction of propagation of the EHG signal. In particular, the area where the EHG signal originates (pacemaker area) was located, for each contraction, by assessing the pacemaker sensor, i.e., the electrode providing the first detection of the burst.

In Fig. 3, the histogram of the pacemaker sensors is reported based on the recorded 110 contractions. The white columns represent the number of contraction for which the sensor was the pacemaker as calculated by delay $\tau$ provided by the adaptive delay estimator. The occurrences of each electrode as pacemaker sensor calculated by prior estimate of the IUP is represented by the sum of the gray and black colored histograms. We represented in black the contractions for which only one pacemaker sensor was found, while we used grey color to represent those contractions where also other electrodes were recognized as pacemaker sensors. The results show that the method based on the prior estimation of the IUP provided more than one pacemaker sensor for a large number of contractions.

By summing the occurrences of the uppermost electrodes $(1,2,3$, and 8$)$ as pacemaker sensors, calculated by the adaptive delay estimator, we deduce that in the $60 \%$ of the cases the contraction started in the upper part of the myometrium, against the $40 \%$ of the other regions.

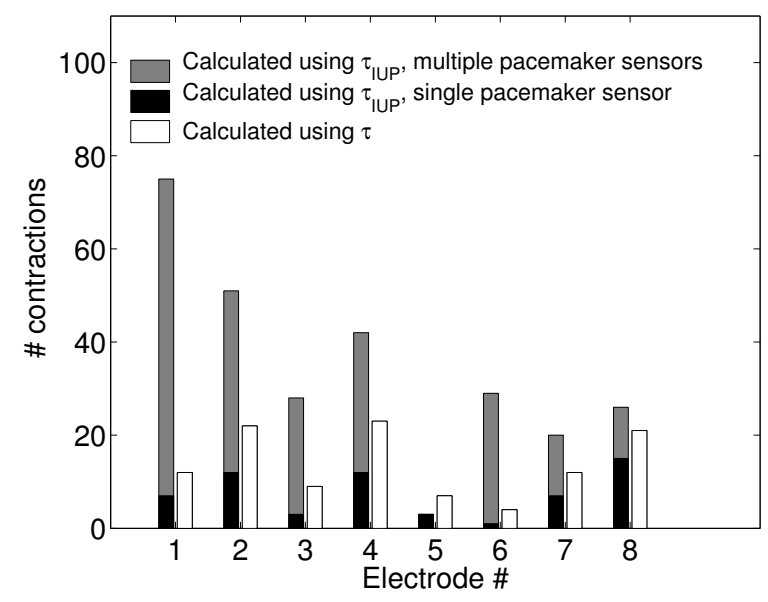

Fig. 3. Histogram of the pacemaker sensor location.

Finally, by analyzing and comparing the delay detected in each channel during a contraction, a study of the global propagation of the EHG signal can be conducted. In Fig. 4(a) and Fig. 4(b), an example of propagation analysis is shown. By interpolating the values of the electrode delays, a spatial grid representing the uterus surface is obtained, where the intensity is proportional to the local delay with respect to the pacemaker sensor. The propagation proceeds from the region with lower intensity towards the regions characterized by a higher intensity. The uterine activation map in Fig. 4(a) was obtained by cross-correlation of the IUP estimates. The electrical signal clearly propagates from the upper right region towards the central-left part of the uterus. In this example, electrodes 1 and 2 have the same detection delay and they are both the pacemaker sensors. From the propagation map, we can also deduce the propagation path on the rear of the uterus. The uterine activation map in Fig. 4(b) was obtained, for the same contraction in Fig. 4(a), by the adaptive delay estimator. Due to the higher temporal resolution of this method, a single pace-maker sensor is recognized and a more accurate description of the propagation path can be obtained.

\section{CONCLUSIONS AND FUTURE WORKS}

In this paper, two methods to calculate the detection delay of EHG signals recorded in a multi-electrode configuration are implemented and compared. These methods are based on the CCF of the estimated IUP and on the adaptive EHG delay estimation in the frequency domain, respectively. The prior estimation of the IUP ensures robustness with respect to poor initial inter-channel correlation, which may affect the accuracy of the adaptive delay estimate. However, this approach has poor temporal resolution. In fact, the delay calculated 


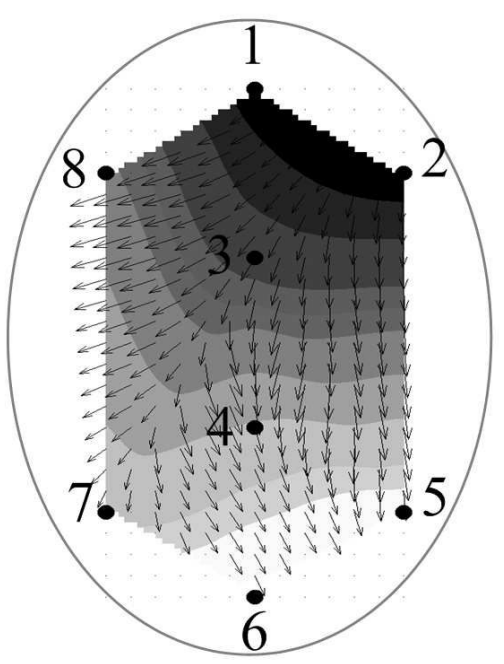

(a)

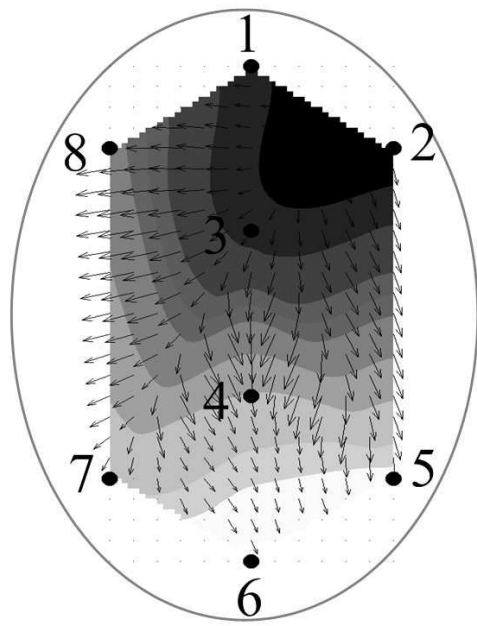

(b)

Fig. 4. Example of visual analysis of EHG signal propagation during a contraction obtained by a) cross-correlation on IUP estimates; b) adaptive delay estimation. The values of the local delays are proportional to the color intensity of the propagation map.

by CCF maximization is limited by the employed sampling rate. Nevertheless, a high sampling rate is not convenient for EHG signals. The Bland-Altman plot demonstrated high agreement between the two methods. However, the average absolute difference between the estimates was higher than the maximum resolution provided by the employed sampling rate.

Despite the low propagation velocities reported in the literature for EHG signals [11], we demonstrated that a high temporal resolution of the calculated delays is essential for the analysis of the direction of propagation, for localization of the pacemaker area, and for possible calculation of the velocity. In particular, the adaptive delay estimator, which provides unlimited resolution at sampling frequencies as low as $4 \mathrm{~Hz}$, resulted in more selective localization of the pacemaker area.

About $60 \%$ of the contractions were first detected by the uppermost electrodes, suggesting a preferred top-down propagation of the myometrial action potential. During labor, this propagation pattern might be associated to the effectiveness of the contractile activity. However, the percentage of the contraction with a top-to-down propagation, as suggested by the histogram of the pacemaker sensor, are lower than expected in a recording covering the last hours of delivery. Nevertheless, it is important to notice that action potentials can originate and propagate also in the rear of the uterus, which cannot be directly monitored, but contributes fundamentally to the mechanical efficiency of a contraction. Therefore, the identification of the pacemaker sensor may provide only partial information on the propagation pattern. Visual tools for the clinical and statistical analysis of EHG propagation, such as the uterine activation maps showed in Fig. 4, can be provided based on the calculation of the interelectrode delay.

Future work will address the development of dedicated signal processing techniques for accurate and high temporal resolution inter-electrode delay estimation for EHG signals. The final objective is supporting further clinical studies and assess the value EHG signal propagation analysis for predicting delivery.

\section{REFERENCES}

[1] J. Linhart, G. Olson, L. Goodrum, T. Rowe, G. Saade, and G. Hankins, "Preterm labor at 32 to 34 weeks gestation: effect of a policy of expectant management on length of gestation." Am J Obstet Gynecol, pp. S178-S179, 1990.

[2] M. Mclean, A. Walters, and R. Smith, "Prediction and early diagnosis of preterm labour: a critical review." Obstet Gynecol Surv, vol. 48, pp. 209-225, 1993.

[3] C. Buhimschi, M. Boyle, and R. Garfield, "Electrical activity of the human uterus during pregnancy as recorded from the abdominal surface." Obstet Gynecol, vol. 90, no. 1, pp. 102-111, Jul 1997.

[4] W. L. Maner, R. E. Garfield, H. Maul, G. Olson, and G. Saade, "Predicting term and preterm delivery with transabdominal uterine electromyography," Obstet Gynecol, vol. 101, pp. 1254-1260, 2003.

[5] M. Doret, R. Bukowski, M. Longo, H. Maul, W. L. Maner, R. E. Garfield, and G. R. Saade, "Uterine electromyography characteristics for early diagnosis of mifepristone-induced preterm labor." Obstet Gynecol, vol. 105, no. 4, pp. 822-830, 2005.

[6] C. Rabotti, M. Mischi, J. O. E. H. van Laar, S. G. Oei, and J. W. M. Bergmans, "Electrohysterographic analysis of uterine contraction propagation with labor progression: a preliminary study," in IEEE Proc on Eng in Med and Biol, 2007, pp. 4135-4138.

[7] R. Vullings, C. Peters, M. Mischi, R. Sluijter, G. Oei, and J. Bergmans, "Artifact reduction in maternal abdominal ECG recordings for fetal ECG estimation," in IEEE Proc on Eng in Med and Biol, 2007, pp. 43-46.

[8] W. L. Maner and R. E. Garfield, "Identification of human term and preterm labor using artificial neural networks on uterine electromyography data," Annals of Biomedical Engineering, vol. 35, no. 3, pp. 465-473, 2007.

[9] J. Lagarias, J. A. Reeds, M. H. Wright, and P. E. Wright, "Convergence properties of the nelder-mead simplex method in low dimensions," SIAM Journal of Optimization, vol. 9, no. 1, pp. 112-147, 1999.

[10] J. M. Bland and D. G. Altman, "Statistical methods for assessing agreement between two methods of clinical measurement." Lancet, vol. 1(8476), pp. 307-310, 1986.

[11] D. Devedeux, C. Marque, S. Mansour, G. Germain, and J. Duchêne, "Uterine emg analysis: a critical review," Am J Obstet Gynecol, vol. 169, no. 6, pp. 1636-1653, Dec. 1993. 